\title{
Barefoot sampling in San Juan de Limay, Nicaragua: remediation of mercury pollution from small scale gold mining tailings
}

Kim H. Esbensen a and Peter W.U. Appel

${ }^{a}$ Research Professor GEUS (Geological Survey of Denmark and Greenland, Copenhagen) and KHE Consulting, Denmark (since August 2015). www.kheconsult.com

${ }^{\mathrm{b}}$ Appel Global, Copenhagen, Denmark. www.appel.global.com

The increasing population on planet Earth has many impacts-one is a strong influence on the amount of mercury released to the environment. Burning of coal in power plants, particularly in China, has tripled several times during the last century and so has the use of coal for cooking for the ever-increasing population of India and elsewhere. These sources account for the second largest release of mercury to the environment. But the worst sinner is the rapidly increasing number of small-scale gold miners in Asia, Africa, Central and South America, who presently provide food on the table for 10 s of millions of households. Small-scale gold miners use vast amounts of mercury for capturing the gold and much of this mercury is released directly to the environment. A large part evaporates to the atmosphere and the rest is transported downstream in rivers ending up in the oceans. The amount of mercury released is phenomenal, an estimated 3000 tons of mercury is released annually by small-scale gold miners alone. A vast proportion enters the food chain in fish and sea mammals, as well as in rice polluted by spillage waters which enters irrigation pathways. Human consumption of polluted fish and/or rice already today has a very severe impact on human health, and this will have even more severe consequences if the current situation is not changed radically. It is of particular concern if mercury-intoxicated women become pregnant because the foetus extracts mercury from the mother. The human foetus is much more sensitive to mercury intoxication and thus has a high risk of being born with brain damage as well as physical disabilities. Over time this will cause reduced intelligence for exposed children in the next generation. This grim outlook has prompted a group of concerned researchers to teach small-scale gold miners to work without the use of mercury and simultaneously to find ways to clean mercury-polluted gold mining tailings, which are one of the main polluting agents. Here we report on one specific part of this endeavour where the Theory of Sampling (TOS) was needed in order to secure reliable estimates of gold and mercury contents in dispersed mining tailings.

\section{Background}

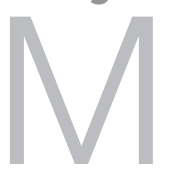

ercury pollution constitutes an environmental time bomb of potentially alarming proportions. The two main sources of global mercury pollution are small-scale gold mining (SSGM) and coal burning in power plants, as well as domestic cooking in developing countries. ${ }^{1}$ The massive release of mercury to our environment will cause a serious global health issue for generations to come; the possibly worst scenario is that humanity will experience a dramatic decrease in intelligence in future generations. Small-scale gold mining is a low-technology, poverty-driven way for many tens of millions of people to provide for their daily needs.

Small scale miners crush and mill gold ore together with mercury. The mercury captures the gold by forming an amalgam. This is subsequently heated in open vessels whereby mercury evaporates and the gold is left behind for economic recovery. This is a technologically simple and very easy processing method that does not require any noticeable investment in equipment and in this way "nothing goes to waste" of the precious gold. Unfortunately, it is the key process element of milling gold ore together with mercury that creates the serious health problem described. During milling a large part of the mercury is ground to small drops called mercury flour. ${ }^{2,3}$ Mercury flour cannot coalesce and can therefore not be recovered by the miners, but ends up in tailings (waste dumps from SSGM operations). This mercury loss is doubly unfortunate-both for the miners and for society. Not only is mercury flour harmful to the environment, but it also constitutes a financial problem since it still contains appreciable amounts of gold that cannot be recovered with the simple methods employed and thus reduces the economic viability of mercurybased SSGM. Over time mercury flour in SSGM tailings will evaporate or gradually be washed into the drainage system, ultimately ending up in the world's oceans from where the evaporated mercury will be distributed over the entire planet. The part of mercury that ends up in rivers, lakes and oceans will be transformed to the compound methylated mercury, or "organic mercury", which readily enters the food chain(s), where it will be bio-magnified, resulting in high concentrations of toxic mercury compounds in toplevel fish and sea mammals. Polluted fish are unfortunately consumed by humansthis is the root cause of the very serious health problems that have been called the impending global mercury disaster. ${ }^{4}$

One principal way to mitigate this mercury disaster is to clean the hundreds of thousands of SSGM tailings containing mercury flour which are littering large parts of South-east Asia, Africa, Central and South America. If an efficient, inexpensive low-tech method can be found, it will benefit not only the global environment and health status of millions, but will at the same time also produce considerable amounts of gold in quantities that may well cover the costs of modified processing methods, and eventually result in more profitability for the SSGM communities.

\section{Barefoot sampling in San Juan de Limay, Nicaragua}

Experiments to extract mercury flour from polluted tailings by alternative, mercury-free 
approaches have been carried out in the Philippines $^{2}$ and are presently being tested in Nicaragua. In Nicaragua, tailings from a number of different SSGM processing sites were subjected to the most advanced alternative recovery process currently available. The experimental tailing lots varied from 4 tons to 15 tons. The first critical step in these experiments was to obtain reliable estimates for the average mercury and gold contents in the tailing heaps available for this experimental campaign; these concentrations are known to be of the order of $2-15 \mathrm{ppm}$. This is no small challenge in a setting where tailings typically are of the order of $\sim 10$ tons, and everything has to be carried out manually.

It is critical to follow the tailing mercury and gold throughout the full multi-stage recovery process and to be able to carry out a complete metallurgical accounting. For this the original $\mathrm{Au}$ and $\mathrm{Hg}$ concentrations in the primary lots are the key information needed, as are the sampling processes employed for dealing with these very low abundances. We here report on barefoot sampling in which application of the principles of the Theory of Sampling (TOS) was de rigeur, but with only DS 3077 and willing, able hands available. For environmental and individual miner health reasons, it is even more important to keep track of the $\mathrm{Hg}$ concentrations at all sampling stages as well, which poses its own specific problems.

\section{Nicaragua field experiment-prospects}

During spring and autumn 2015, feasibility tests were out carried in Nicaragua to estimate the efficiency of extracting mercury flour from SSGM tailings. To the degree this is feasible, and to the degree it can be successfully recovered at a sufficiently high recovery rate $(\sim 75 \%)$, this will be a significant driver to allow SSGM collectives to accept the alternative process. ${ }^{\dagger}$

Investment capital for this type of local mining reclaiming is available a.o. from developed nations' development funds, and there is also direct commercial potential. The alternative process will be profitable, at assumed recovery rates larger than

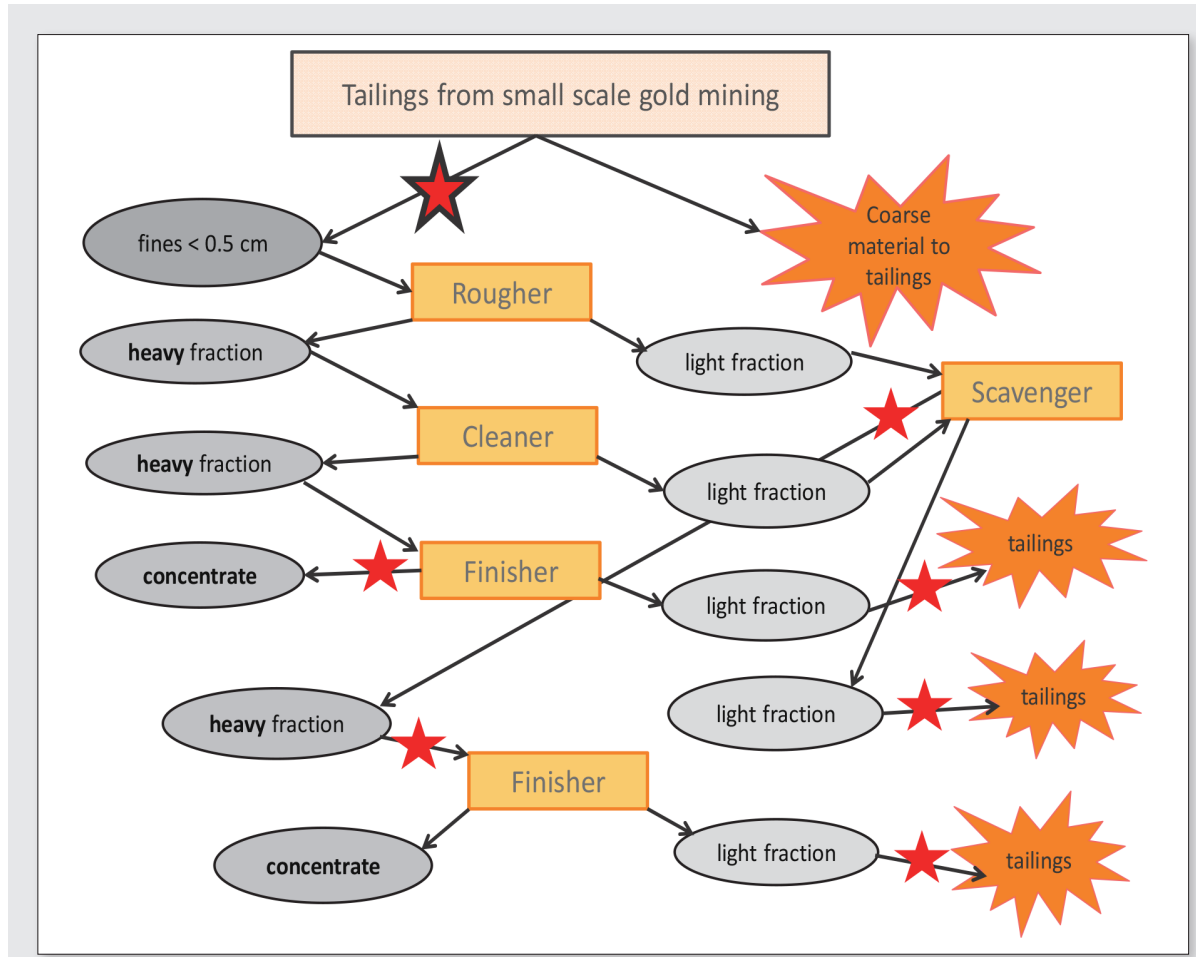

Figure 1. Schematic overview of the SSGM tailing reclaiming process with sampling points for the complete pilot study (small red stars) and the present experiment (large red star with black edge).
$75 \%$, as long as tailing concentrations are above $3 \mathrm{ppm}$. The same process will remedy the otherwise continuing SSGM tailings pollution-a double whopper-and all essentially with barefoot technology!

\section{Field experiment design and sampling requirements (TOS)} Tailings for the experiments were gathered from five different SSGM processing sites. The tailings were selected to represent different types of gold mineralisations and thus different general compositions with presumably differing processabilities, and milling efficiency was indeed observed to vary widely locally. The overall mercury contents would therefore be expected to vary significantly between different tailings (corroborated by the analytical results, see Table 1). Tailing lot masses varied from 4 tons to 21 tons with an average of $\sim 10$ tons.

The crucial first step is to establish the average mercury content of each tailing lot with absolutely no primary sampling bias allowed because of the ultra-low grade levels present. For this reason, sampling
${ }^{\dagger}$ There exists an alternative mercury-free gold extraction method, which is gradually gaining momentum in South-east Asia. This approach uses gravitational separation to produce a gold concentrate, which is subsequently smelted by using the environmentally benign chemical compound borax. ${ }^{2,3,5,6} \mathrm{An}$ introduction to this approach can be seen on an educational video here: https://www.youtube.com/ watch? $\mathrm{V}=\mathrm{X} 6 \mathrm{SawjOHyFO}$. expertise in the form of the TOS was called upon. It is equally important to be vigilant with respect to the representativity of all subsequent field and laboratory massreduction steps. From original tailing size to analytical mass, sampling rates are of the order of $1: 10^{7}$. The principles of TOS have to be upheld scrupulously along the entire lot-to-analysis pathway. While this is trivial in most scientific, technological and industrial contexts in the developed world, the present project poses a highly challenging twist: almost everything must be carried out manually - which calls for barefoot field sampling (for the first two stages), but in the subsequent laboratory mass-reduction stages some innovative approaches were also called upon, as described below.

We welcome this challenge-how better to contribute to helping tens of millions of SSGM families with a life-threatening mercury danger?

\section{SSGM tailing recovery process - a brief}

Tailings were scooped into a drum, which selects and discard $>0.5 \mathrm{~mm}$ material. The resulting fines are directed into a train of three spiral concentrators, which separate heavy from light minerals. These are termed "Rougher", "Cleaner" and "Finisher", respectively, in Figure 1. The heavy fraction 


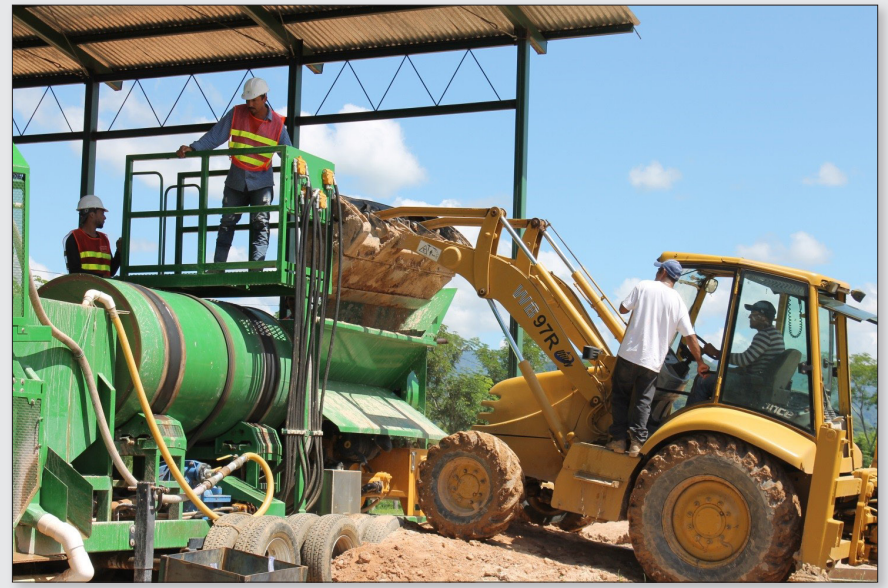

Figure 2. First stage in the SSGM tailing reclaiming process feasibility project, initial particle size screening.

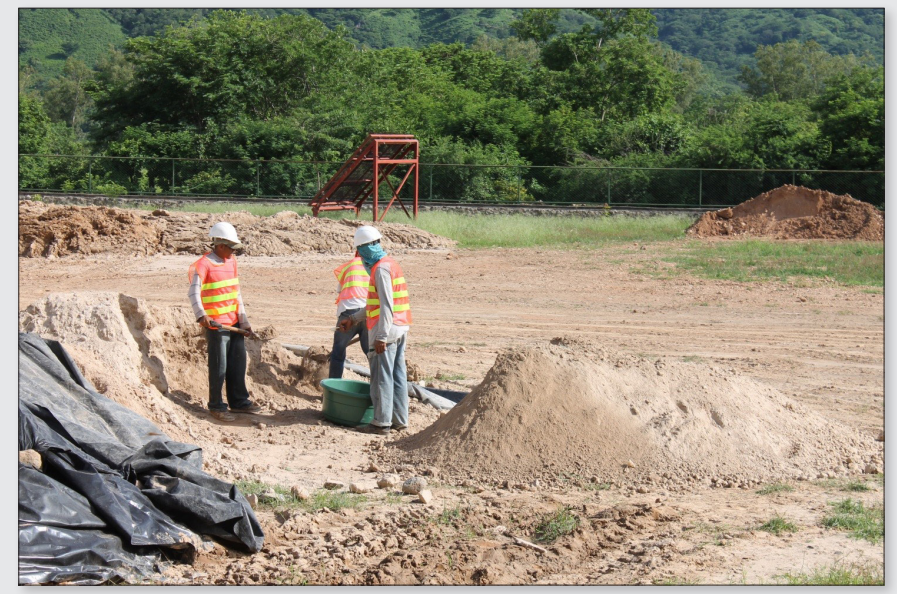

Figure 3. Halfway through the intensive task of moving a complete original lot one shovel at the time, taking great care to extract an increment from each, as detailed in Figure 4. from each spiral is directed to the next spiral. The light fraction from "Rougher" and "Cleaner" is directed to a centrifuge, termed "Scavenger". The light material from here is directed to tailings while the heavy fraction is directed to the "Finisher". The heavy fraction from the "Finisher" is directed to a stack of copper amalgamated plates "Peter plates", 2 which finally capture the mercury flour and free gold particles to be reclaimed. Figure 2 shows the first stage of the full feasibility study (drum loading for initial particle size screening). Below we are exclusively interested the critical primary sampling from the original tailings: how to get a documentable representative analytical estimate of the average gold grade?

\section{Primary lot sampling - the crucial stage}

Manipulating lots of the size of $5-15$ tons is usually not a problem when the appropriate industrial equipment is at hand, e.g. front-loaders, bobcats or the like-of which there most emphatically are none available to very poor artesian mining collectives. But able hands, picks and shovels are in abundance. It was decided to follow the principle: "move the original lot $10 \mathrm{~m}$ to the right" and perform process sampling along this 1-D transportation stage. Thus each tailing dump was transported manually, one shovel-full after another in order to facilitate sampling, Figure 3.

Figure 4 shows this primary composite sampling in extremis: the material in each shovel-blade (approx. $5 \mathrm{~kg}$ ) is intercepted by a scoop of approximately weight $100 \mathrm{~g}$. For an average 10-ton primary lot size, this translates into 2000 increments (each of $\sim 100 \mathrm{~g}$ ), by all standards an overwhelming coverage of each original lot with a solid guarantee for compliance with the Fundamental Sampling Principle (FSP). The resulting composite primary sample weighs $\sim 200 \mathrm{~kg}$. This material was subjected to forceful mixing before further sub-sampling, based on the abundant man-power available.

Figure 5 shows how the next sub-sampling stage was executed: each $200 \mathrm{~kg}$ primary sample was passed through a riffle splitter, in a series of 50/50 split sessions until the sub-sample mass had been reduced to $\sim 1-2 \mathrm{~kg}$, which was the sample size subsequently transported to GEUS, Denmark for further processing and preparation for analysis.

\section{Slurry sub-sampling in the laboratory}

After processing all primary tailings in the manner illustrated, quantitative analysis was carried out on a selected set of seven primary samples (project financing was at the time of the analysis also at a decidedly "barefoot" level). These samples were not easy to process, however, as they were all slurries and with very different $\mathrm{Au}$ and $\mathrm{Hg}$ contents. Slurry sampling is not easy under any circumstance, but especially not when

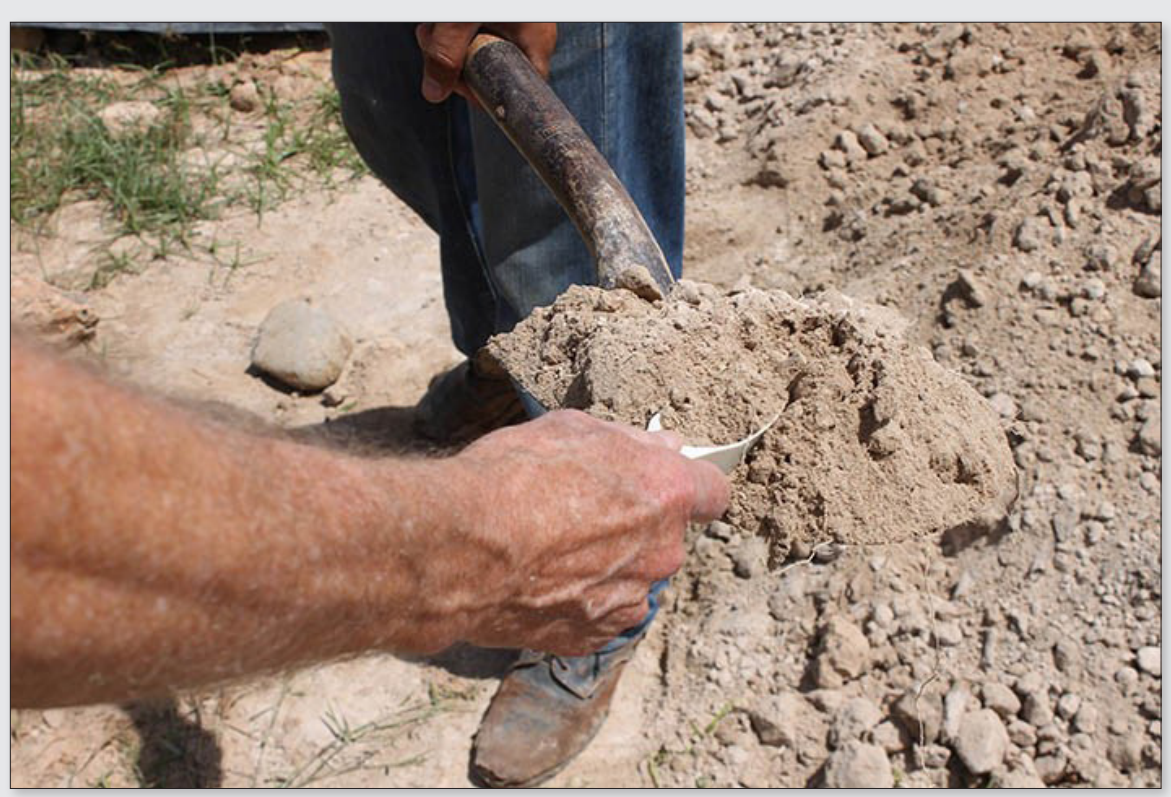

Figure 4. Incremental sampling from each shovel used to transport all original lots, see Figure 3. 


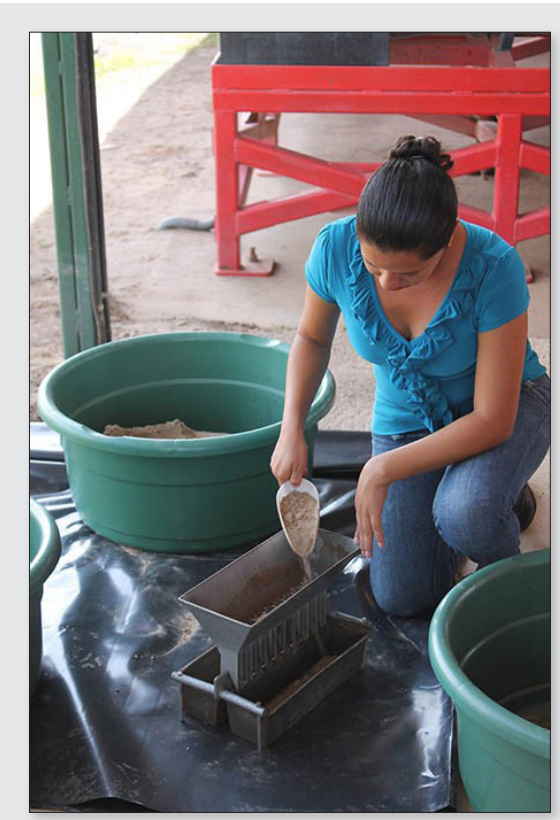

Figure 5. Loading the project riffle splitter (kindly provided by GEUS). Sub-sampling is made effective by the fact that the sample to be split does not need to be split all in one, but can be subjected to riffle-splitting in an intermittent loading process.

stringent counter-volatility demands are to be upheld. Also, sub-sampling, although here carried out in a well-equipped laboratory (GEUS, Copenhagen), had to be performed with procedures that potentially can be carried out under the relevant ambient field conditions in Nicaragua.

Due to the severe risk of segregation (free $\mathrm{Au}$ particles, mercury flour), handling the slurry samples became a critical issue, not easily tackled with the standard riffle splitters at hand. It is critical to counteract anyand-all segregation effects present in the sub-sampling procedure employed (while these effects may be small, intermediate or large, they are never absent and the only responsible approach is to assume such effects are always significantly present). For this reason, conventional riffle splitting could not be used. A better way was required, illustrated in Figure 6.

A novel twist had to devised: after vigorous and extensive mixing, the entire $1-2 \mathrm{~kg}$ slurry samples, which came in tightly sealed but otherwise conventional plastic bottles or bags, were stored in a freezer $\left(-16^{\circ} \mathrm{C}\right)$ for $24 \mathrm{~h}$, sufficient for the entire content to freeze solid. The "splitting" was then effectuated as a two- or three-step longitudinal sectioning of the solid bottle or bag content, see Figures 7 and 8. In this way any residual segregation affecting the vertical container

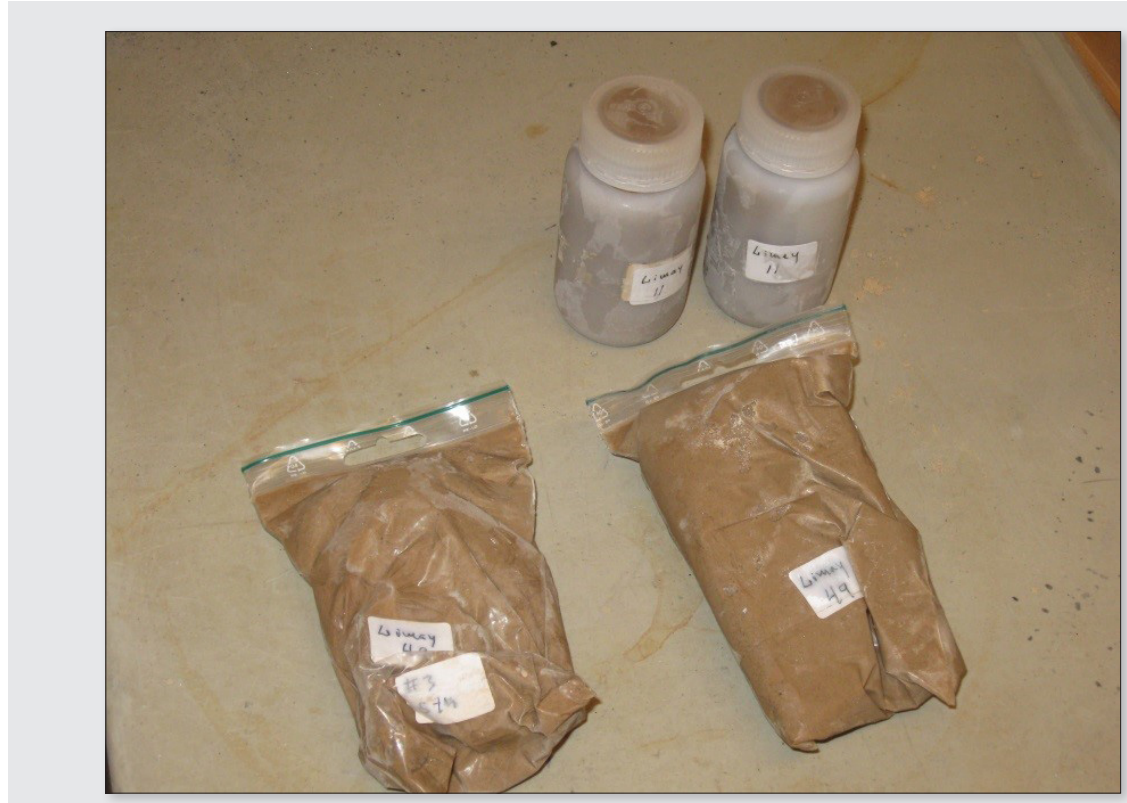

Figure 6. Two types of slurry sample containers as received from Nicaragua, plastic bottles and bags. The photo shows the frozen versions after $24 \mathrm{~h}$ in a freezer at $-16^{\circ} \mathrm{C}$, ready for sub-sampling, see Figures 7-8.

contents in their slurry state was sampled in a fully representative fashion, while maintaining quite effective sampling rates of the order of $1: 10$ at this stage. This sub-sampling technique is only dependent on the cohesiveness of the frozen solid w.r.t. the thickness of the slice cut with the diamond saw (and subsequently with a hobby knife).

From the primary sampling stage all the way through the penultimate sample shipped off to the analytical lab, the critical success factor was counteracting segregation. Even the commercial, accredited ACTlab analytical laboratory was directed to document the in-house sub-sampling employed with reference to DS $3077^{8, \neq}$

All 34 final sub-samples were of analytical mass $\sim 12 \mathrm{~g}$ when shipped off for multi-element analysis (standard economic geology element suite plus $\mathrm{Au}$ and $\mathrm{Hg}$ ) at Actlabs Labs, Canada.

${ }^{\ddagger}$ Appendix 1 quotes our directions to ensure full disclosure of the in-house laboratory procedures w.r.t. sub-sampling. At the present extremely low average gold grade there is every possibility that the lot-to-aliquot integrity can be significantly affected by mindless grab sampling (using a spatula) even at this ultimate sampling stage. Indeed the size of gold particles and mercury flour drops may be fatally mis-sampled with a spatula.

\section{Sampling perspective}

The original lots were on average of a size corresponding to 10 tons. The average assumed Au-grade in the mine tailings was of the order of 2-15ppm, based on the best local mining experience available. The lot material, earlier mining tailings, is crushed to an average grain size diameter of $50 \mu \mathrm{m}$, but the tailings have never been subjected to mixing or blending to any extent. It follows that such very low-grade gold concentration carriers (flakes, particles) must be present in an exceedingly irregular spatially heterogeneous distribution pattern, i.e. for the present study the lot distributional heterogeneity is extreme. It would be close to a miracle if standard haphazard scooping of a small primary sample from 10 tons, archetype grab sampling, could ever be representative. ${ }^{8}$ Only a very thorough composite sampling can be accepted. In order to meet these hard demands, the "field-to-analysis" pathway consisted of i) primary sampling (extremely effective composite sampling with a sampling rate of $1: 10^{4+}$ ); ii) field mass-reduction (riffle splitting, sampling rate $\sim 1: 100$ ); longitudinal diamond saw cutting of premixed vertical slices (1:10); in-house aliquoting sub-sampling $(\sim 1: 10)$. The complete sampling pathway mass reduction thus spanned seven orders of magnitude (mass/mass). All stages were carefully designed and scrupulously performed in 

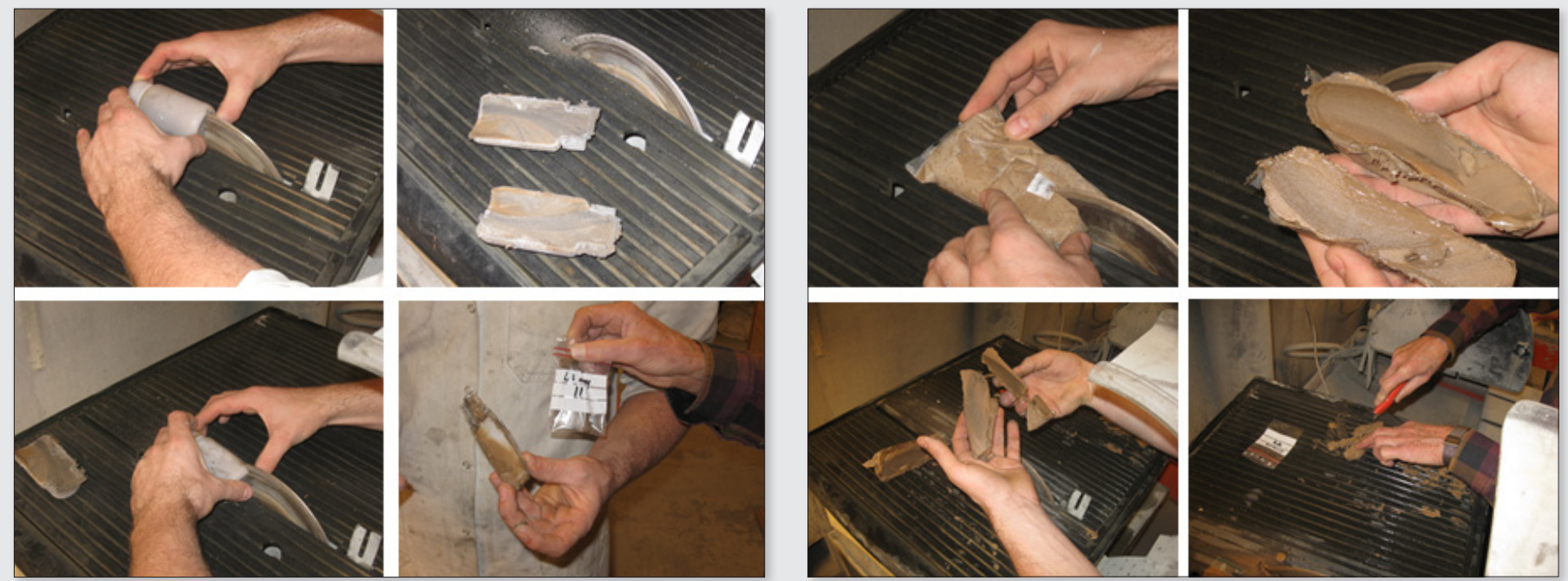

Figure 7. Two-stage mass reduction of frozen sample bottles. First cutting is a 50/50 split, followed by a further slice of one of the randomly selected half cores produced, resulting in a 10-15\% final sub-sample mass, which is guaranteed to be representative of the original container content in the vertical dimension irrespective of the degree of possible residual segrega-

tion present.

Figure 8. Three-stage mass reduction of frozen sample bag content. All cuts are vertical slices again resulting in a 10-15\% final sub-sample mass, which is guaranteed to be representative of the original container content in the vertical dimension irrespective of the degree of residual segregation present.

accordance with TOS' every principle for representative sampling to a degree only rarely deployed within the geosciences, while at the same time exclusively only relying on manual processes. ${ }^{7,9}$ The term "barefoot sampling" appears apt.

\section{Project results - preliminary findings}

Table 1 shows the analytical results from the primary samples representing the seven mining tailings addressed in the sampling feasibility pilot study.

\section{Discussion}

In industrial contexts, an average gold grade of above $\sim 5 \mathrm{ppm}$ is considered profitable; in the SSGM community, 3ppm (and above) is eminently interesting. In this view the values revealed in Table 1 show the inefficiency of traditional gold extraction using mercury. There is therefore an important incentive to address these types of tailings. There are two options:

1) extraction of gold from original deposits using the alternative mercury-free procedure and/or

2) re-process SSGM tailings, also based on the alternative approach.

Re. 1) Teaching small-scale miners mercury-free extraction procedures constitutes an important objective-which will result in an increased profitability of at least $50 \%$, while simultaneously contributing significantly to solving the global mercury poisoning threat. ${ }^{2}$
Re. 2) This will directly reduce the global mercury pollution - while at the same time being able to score economically from a source never mined optimally before, SSGM tailings. Some operators have run these tailings through a cyanide leaching process, which has its own, severe environmental problems of course.

\section{Conclusions}

This work originated as part of a global mercury pollution reduction endeavour to which SSGM is the major contributor. The mercury is hosted in tailings as tiny droplets, which are difficult/impossible to recover. The present feasibility project, financed by the Danish Ministry of Environment and Food, focuses on methods for mercury cleaning

Table 1

\begin{tabular}{|c|c|c|c|c|c|c|c|c|c|}
\hline Analyte & $\mathrm{Au}$ & $\mathrm{Ag}$ & $\mathrm{Hg}$ & $\mathrm{Cu}$ & $\mathrm{Pb}$ & $\mathrm{Zn}$ & As & Se & Sb \\
\hline Unit & ppm & ppm & ppm & ppm & ppm & ppm & ppm & ppm & ppm \\
\hline Detection limit & 0.03 & 0.5 & 10 & 0.03 & 0.03 & 0.3 & 0.05 & 0.05 & 0.05 \\
\hline Analysis method & FA-GRA & ICP-MS & ICP-OES & ICP-MS & ICP-MS & ICP-MS & ICP-MS & ICP-MS & ICP-MS \\
\hline Limay1 & 13 & 7.1 & 10 & 45.1 & 54.2 & 50.2 & 10.4 & 4.89 & 4.78 \\
\hline Limay13 & 4.99 & 121 & 20 & 332 & 592 & 88.2 & 96.1 & 9.37 & 4.2 \\
\hline Limay23 & 4.41 & 28.2 & 20 & 317 & 241 & 55.7 & 8.03 & 7.12 & 2.62 \\
\hline Limay30 & 1.15 & 7 & 10 & 151 & 148 & 165 & 58 & 6.15 & 2.86 \\
\hline Limay36 & 9.87 & 8.9 & 10 & 46.9 & 65 & 61.6 & 6.74 & 6.14 & 4.13 \\
\hline Limay44 & 13.5 & 10.5 & 10 & 34.1 & 67.7 & 51.9 & 7.58 & 5.88 & 3.44 \\
\hline Limay51 & 3.95 & 222 & 40 & 176 & 709 & 197 & 224 & 7.71 & 7.36 \\
\hline
\end{tabular}


with the aim of two potential bonuses for the SSGM community, and for the world, one environmental, the other economic.

In this context, a need for careful primary sampling was identified. Global SSGM tailings, destined for an improved, non-toxic reclaiming process, need to be characterised with extraordinary focus on reliable estimates of average grades for $\mathrm{Au}$ and $\mathrm{Hg}$.

For this purpose, the TOS was invoked which had to be applied subject to stringent "barefoot" technology requirements. Amongst others, use was made of extraordinarily intensive composite primary sampling in full compliance with the FSP. The project also developed a "freeze-drying" technique for sampling "difficult" slurry samples with severe gold segregation and mercury integrity issues. As described and illustrated, these TOS tasks were satisfactorily resolved. There is likely a carrying-over potential for the freeze-dried sub-sampling procedure to other similar types of slurry material.

\section{Appendix 1}

"These samples originate from a study of low (to very low) Au and $\mathrm{Hg}$ concentration in mine tailings and tailing dumps (estimated 2-15 ppm), implying a highly irregular distribution of elemental micro-Au flakes/fragments in the 15 ton original tailing dump. The project has invested a considerable effort in arriving at the seven sample flasks supplied (masses $~ 30 \mathrm{~g}$ ) with outmost care in using Theory of Sampling compliant primary and secondary sub-sampling throughout, as documented in DS 2077 (2013). It is critical that also the final mass-reduction needed for ACTLABS to extract the precise analytical aliquot mass/volume are fully representative, i.e. extracting the aliquot mass from the sample flasks supplied by spatula is unacceptable. We ask ACTLABS to follow one of the recommended procedures in Petersen et al. (2004), Esbensen \& JuliusPetersen (2009). Because this project is a method-development feasibility study in which sampling, handling and analysis are of equal importance, we ask ACTLABS to supply a complete documentation of the inhouse sub-sampling employed."

\section{Acknowledgements}

The first author notes with satisfaction GEUS' involvement in this type of development aid project and sends a big Thank You to student assistant Toke Hvenegaard for superb diamond saw cutting and safety diligence in the geo-lab.

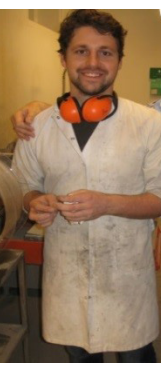

The second author is grateful for the support provided by the MUDP programme of the Danish Ministry of Environment and Food and to Encinal Resources S.A. for allowing access to their processing plant in Nicaragua. Partners in the project are Institute of Metallurgy at Danish Technical University, Elplatek A/S, Denmark and Oro Industries, California.

\section{References}

1. AMAP/UNEP, Technical Background Report for the Global Mercury Assessment 2013. Arctic Monitoring and Assessment Programme, Oslo, Norway/UNEP Chemicals Branch, Geneva, Switzerland (2013).

2. P.W.U Appel, L.D. Na-Oy, Y. Hatsukawa, T. Osawa, J. Kystol and L.L. Sørensen, "Cleaning mercury polluted mine tailings in the Philippines", Danmarks. Grønlands. Geol. Unders. Rap. 2011/127 (2011).
3. P.W.U. Appel and D.L. Na-Oy, "Mercury-free gold extraction using borax for small-scale gold miners", J. Environ. Protect. 5, 493499 (2014). doi: https://doi.org/10.4236/ jep.2014.56052

4. S. Bose-O'Reilly, B. Lettmeier, R.M. Gohte, C. Beinhoff, U. Siebert and G. Drasch, "Mercury as a serious health hazard for children in gold mining", Environ. Res. 107, 89-97 (2014). doi: https://doi.org/10.1016/j. envres.2008.01.009

5. P.W.U. Appel, A. Andersen, L.D. Na-Oy and R. Onos, "Introduction of mercury-free gold extraction methods to medium-scale miners and education of health care providers to reduce the use of mercury in Sorata, Bolivia", J. Health Pollut. 5(9), 12-17 (2015). doi: https://doi.org/10.5696/2156-9614-5-9.12

6. A.R. Scialli, J.P. Bonde, I. Brüske-Hohlfeld, B.D. Culver, Y. Li and F.M. Sullivan, "An overview of male reproductive studies of boron with an emphasis on studies of highly exposed Chinese workers", Reproduct. Toxicol. 29, 10-24 (2010). doi: https://doi. org/10.1016/j.reprotox.2009.10.006

7. K.H. Esbensen and L.P. Julius, "Representative sampling, data quality, validation - a necessary trinity in chemometrics", in Comprehensive Chemometrics, Ed by S. Brown, R. Tauler and R. Walczak. Wiley Major Reference Works, Vol. 4, pp. 1-20 (2009). doi: https://doi.org/10.1016/B978-0444527011.00088-0

8. DS 3077, DS 3077. Representative Sampling-Horizontal Standard. Danish Standards (2013), www.ds.dk

9. K.H. Esbensen and C. Wagner, "Theory of sampling versus measurement uncertainty (MU) - A call for integration", Trends Anal. Chem. 57, 93-106 (2014). doi: https://doi. org/10.1016/j.trac.2014.02.007

\section{Be part of the next issue of TOS forum!}

We welcome contributions to TOS forum: articles, letters, comment, news or news of PhD projects for the PhD Presentations column.

TOS forum Editor, Kim Esbensen, would be pleased to discuss any ideas you may have and to receive your contributions.

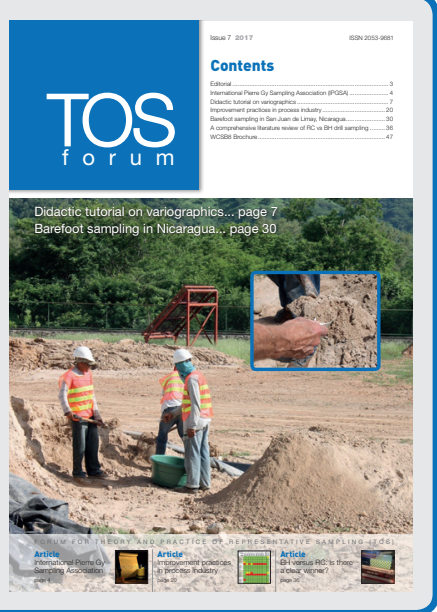

